\title{
Entwicklung und Struktur der Epidermisaussenwand bei einigen Angiospermenblättern
}

\section{Doctoral Thesis}

Author(s):

Bolliger, Rudolf

Publication date:

1959

Permanent link:

https://doi.org/10.3929/ethz-a-000089057

Rights / license:

In Copyright - Non-Commercial Use Permitted 
Prom.-Nr. 2890

\title{
Entwicklung und Struktur der Epidermisaussenwand bei einigen Angiospermenblättern
}

\author{
Von der \\ EIDGENÖSSISCHEN \\ TECHNISCHEN HOCHSCHULE IN ZÜRICH \\ zur Erlangung der Würde eines Doktors \\ der Naturwissenschaften \\ genehmigte \\ PROMOTIONSARBEIT \\ vorgelegt von \\ RUDOLF BOLLIGER \\ dipl. Naturwissenschafter \\ von Holziken $(A G)$
}

Referent: Herr Prof. Dr. A. Frey-WyssLing

Korreferent: Herr Prof. Dr. K. MÜhlethale R 


\title{
Entwicklung und Struktur der Epidermisaussenwand bei einigen Angiospermenblättern
}

\author{
R. Bolliger \\ Institut für Allgemeine Botanik der Eidgenössischen Technischen Hochschule, Zürich \\ Eingegangen am 18. März 1959
}

\begin{abstract}
An Blättern von Philodendron scandens wurde die Entwicklung von Kutikula und Kutikularschicht während des Wachstums untersucht. Die Resultate erlauben Vergleiche mit der Epidermisaussenwand anderer Angiospermenblätter.

Die aufbauenden Elemente der Kutikula, ungesättigte Fettsäuren, können in $80-200 \AA$ grossen Lipoidtröpfchen nachgewiesen werden. Die Wanderung dieser Stoffe durch die zellulosische Zellwand verläuft völlig passiv, d.h. durch Diffusion und den kutikulären Transpirationsstrom. Sie diffundieren als KolIoidteilchen (Abb. 11). Ektodesmen konnten keine gefunden werden. Kutikula und Kutikularschicht entstehen nacheinander und die betreffenden Bausteine scheinen sich voneinander zu unterscheiden.

Die zellulosehaltigen Membranen entstehen nach dem Prinzip des MultiNetz-Wachstums (24). Ein vom Appositionswachstum abweichender Mechanismus konnte nicht nachgewiesen werden.

UV-Absorptionsmessungen an unbehandelten Epidermisaussenwänden ergaben, dass die Kutikula als physiologisch wirksamer Strahlenschutz nicht in Frage kommt, auch nicht bei Hochgebirgspflanzen. Die Untersuchungen mit ultraviolettem Licht erlaubten auch einige Rückschlüsse auf den Chemismus des Kutins, das ungesättigte Komponenten enthalten muss.
\end{abstract}

Die Oberhaut oder Epidermis schliesst als schützende Hülle die oberirdische Pflanze nach aussen $a b$. Sie ist allgemein einschichtig, nur selten, bei Xerophyten, von einer Hypodermis unterlagert, und bildet meistens ein derbes, gut zusammenhängendes Häutchen, das dem darunterliegenden Gewebe Schutz bietet. Die Schutzfunktion kann die Epidermis vor allem dank ihrer verdickten, hydrophoben Aussenwand erfüllen, und diese hängt in ihrer Funktionstüchtigkeit vom mikroskopischen und submikroskopischen Bau der Wand ab. Ihre Aufgabe besteht vor allem darin, das Mesophyll vor einem allzustarken Feuchtigkeitsverlust zu schützen. Dies vermögen die Aussenwände der Epidermiszellen deshalb zu erfüllen, weil sie relativ grosse Mengen von Lipoiden: Kutin und Wachse enthalten. Diese Stoffe treten in der Epidermis in Form von parallel zur Oberfläche gelegenen Schichten auf, die bei Xerophyten auch in der darunterliegenden Hypodermis angetroffen werden können. 